\title{
ATUAÇÃO DO PROFISSIONAL DA INFORMAÇÃO NA ORGANIZAÇÃO DO CONHECIMENTO NA WEB DE DADOS
}

\author{
Francisco Carlos Paletta ${ }^{1}$, Armando Manuel Barreiros Malheiro da Silva ${ }^{2}$ \\ ${ }^{1}$ Universidade de São Paulo, http://orcid.org/0000-0002-4112-5198, fcpaletta@usp.br, \\ ${ }^{2}$ Universidade do Porto, http://orcid.org/0000-0003-0491-3758, malheiro@letras.up.pt
}

\begin{abstract}
Resumo: A inclusão digital está também relacionada ao desenvolvimento nos indivíduos de habilidades e competências para acesso, uso e disseminação eficiente da informação. Além disso, a evolução contínua tecnológica, tal como a emergência da Web semântica, leva a novos perfis e demandas do profissional da informação. A organização e gestão da informação com grandes coleções de materiais necessitam reavaliar constantemente a identidade e propósitos principais de seus usuários. Bibliotecas estão desenvolvendo continuamente processos e serviços de forma a explorar os benefícios da Web Semântica. Neste trabalho, discutimos as principais tendências nesse campo, em especial aquelas que influenciam o contexto da Web de Dados.
\end{abstract}

Palavras-chave: Organização da Informação, Gestão do Conhecimento, Web de Dados, Era Digital, Profissional da Informação.

Abstract: Digital inclusion is also related to the development of individuals in skills and competencies to access, use and efficiently dissemination of information. Besides, continuous technological developments such as the emergence of the semantic Web, leads to new professional profiles and demands. The design and management of information systems with large collections of materials need to constantly re-evaluate the identity of its users and their main purposes. Libraries are continually developing processes and services to exploit the benefits of the Semantic Web, for instance. In this paper, we discuss the main trends in this field, especially those that influence the context of the Web of Data.

Keywords: Information Organization, Knowledge Management, Web of Data, Digital Age, Information Professional.

\section{INTRODUÇÃO}

A Web é uma rede com conteúdos interligados através de documentos de hipertexto. Seu mapeamento é possível por processos de análise e coleta sucessiva de páginas de conteúdo a partir de um conjunto de localizações de documentos previamente conhecido. Tais buscas são feitas de forma automática por programas de computador normalmente denominados crawlers, coletores ou batedores. Mesmo neste mapeamento automático, o conjunto inicialmente assumido de localizações a partir do qual a pesquisa é feita influencia o resultado obtido. Além disso, nem toda a Web está interligada: existem muitos subconjuntos de documentos interligados entre si, de tamanhos variados, sem ligação com o restante da rede (ou seja, "ilhas" de informação). O primeiro princípio da Web, proposto pelo Consórcio World Wide Web W3C Brasil afirma (W3C, 2011): 


\title{
III CONGRESSO ISKO Espanha - Portugal
}

\begin{abstract}
"O principal valor da Web é o social. Mais do que tecnológico, este é um ambiente de comunicação humana, de transações comerciais, de oportunidades para compartilhar conhecimentos e, para ser um ambiente universal, deve estar disponível para todas as pessoas, independentemente dos equipamentos e softwares que utilizem, principalmente da cultura em que se inserem, da localização geográfica, das habilidades físicas ou mentais, das condições socioeconômicas ou de instrução".
\end{abstract}

A universalidade da Web só pode ser garantida e aprofundada com um modelo de governança democrático e pluralista que tenha foco no acesso por todos e na sua própria evolução tecnológica (CGI.br, 2011).

A temática da inclusão digital vem sendo tratada desde os anos 90 como a necessidade de permitir o acesso a computadores e ferramentas de TICs (Tecnologia da Informação e Comunicação). No entanto, novas proposições têm tratado da inclusão não apenas no acesso às ferramentas digitais, mas também nos aspectos relativos ao acesso e uso dos conteúdos distribuídos. Promover a inclusão digital e por consequência a inclusão social não significa apenas promover universalidade das ferramentas de TICs, mas possibilitar seu uso de forma crítica, estimulando o aperfeiçoamento das potencialidades informativas e cognitivas e atividades cidadãs. A preocupação deixa de ser o aprendizado de ferramentas básicas de navegação na WEB e desloca-se para as diferentes formas de coleta e de produção do conhecimento. Tornam-se centrais questões de inclusão social através da inclusão digital e das práticas sociais e educacionais vigentes (Passarelli 2012).

A expressão Competência Informacional, ou Information Literacy, tem suas origens no estudo da sociedade da informação, e está ligada à necessidade de se desenvolver nos indivíduos aptidões sobre habilidades e competências relacionadas ao acesso, uso e disseminação da informação, objetivando fazer uso desta de forma ética e eficiente, para que o ser humano através de seu intelecto e processo cognitivo possa produzir novo conhecimento (Campello 2003).

Literacia informacional tem constituído, nos últimos anos, novo campo de pesquisa que interessa à educação, à ciência da informação e às ciências cognitivas. $\mathrm{O}$ desafio está em inicialmente aprender a utilização básica dos recursos tecnológicos - literacia digital - e a seguir apropriar-se dos mesmos para gerar novos conhecimentos - literacia informacional. No contexto da literacia informacional, os desafios são múltiplos: a dificuldade das bibliotecas universitárias, com suas coleções caríssimas, em concorrer com acervos digitais; as bibliotecas virtuais e os motores de busca oferecendo informações de forma imediata e a custo zero; a desconstrução do conceito de autor individual e a emergência dos coletivos digitais. Os novos contornos da sociedade em rede têm constituído objeto de estudo e pesquisa para profissionais das mais diversas áreas de atuação.

Esta pesquisa de caráter qualitativo e exploratório tem como objetivo proporcionar uma reflexão sobre a atuação do profissional da informação na organização do conhecimento na Web de Dados, com vistas a torná-lo mais explícito ou a construir hipóteses. 


\section{CONGRESSO ISKO Espanha - Portugal}

\section{O PROFISSIONAL DA INFORMAÇÃO E A ERA DIGITAL}

No contexto mundial definido pela globalização e pela constante mudança tecnológica, o conhecimento tornou-se a principal riqueza das nações, pode também constituir o principal fator de desigualdade nas sociedades atuais. A Sociedade da Informação é a pedra angular das Sociedades do Conhecimento. O conceito de "sociedade da informação", relacionado à ideia da inovação tecnológica, caracteriza-se pelo rápido crescimento da informação disponibilizada e as mudanças ocasionadas pela tecnologia usada para gerar, disseminar, acessar e usar a informação (Melo 2007).

Por outro lado, o conceito de "sociedade do conhecimento" inclui uma dimensão de transformação social, cultural, econômica, política e institucional, assim como uma perspectiva mais pluralista e de desenvolvimento, expressando a complexidade e o dinamismo das mudanças que estão ocorrendo. $\mathrm{O}$ conhecimento não só é importante para o crescimento econômico, mas também para fortalecer e desenvolver todos os setores da sociedade. De acordo com Unesco (2017):

"O conhecimento e a informação têm impacto significativo na vida das pessoas. O compartilhamento de conhecimento e informação, particularmente por meio das tecnologias de informação e comunicação (TIC), tem o poder de transformar economias e sociedades... as sociedades do conhecimento devem ser construídas sobre quatro pilares: respeito às diversidades cultural e linguística; liberdade de expressão; acesso universal à informação e ao conhecimento; e educação de qualidade para todos".

A noção de fácil acesso à informação propiciado por avanços da tecnologia de redes de computadores e de telecomunicações criou uma noção errônea do imperativo tecnológico como resposta às deficiências comunicacionais e educacionais da humanidade. O conhecimento e o uso deste ferramental tecnológico são essenciais nos dias de hoje, porém é preciso considerar que por si só a tecnologia não leva à comunicação e à educação. A competência informacional está fortemente relacionada ao processo de interiorização de conhecimentos, habilidades e valores ligados à informação e ao aprendizado. Em um contexto mais prático, representa um conjunto de habilidades necessárias para localizar, interpretar, analisar, sintetizar, avaliar e comunicar a informação em diferentes ferramentas e suportes (Dudziak 2001; 2003).

Indivíduos têm seus estoques de informação e estes são usados para compreender as informações externas e as diferentes situações em que se encontram em dado momento. O comportamento de busca e uso de informação são modelados, do ponto de vista epistemológico, pelas características cognitivas do indivíduo e por fatores que geram o encontro do usuário com os sistemas de informação ou as consequências de tal confronto. A importância do aspecto cognitivo para o comportamento de busca de informação é apresentada por Kuklthau (2008):

"Vários estudos apoiaram a noção de busca de informação como um processo de construção do conhecimento com diferentes estágios cognitivos e afetivos (Cole 1997; George et al., 2006; Harada 2002; Pitts 1995; Tang e Salomão 1998; Serola e Vakkari 2005; Vakkari 2001; Vakkari et al., 2003, Vakkari e Hakala 2000, Wang e Soergel 1998, Yang, 1997). O modelo do Processo de Pesquisa de Informação muitas vezes causa ansiedade e incerteza ........ estudos confirmaram essa noção de interação entre os fatores cognitivos, afetivos e comportamentais na busca da informação (Bilal 2000; 2001; 2002; Bilal e Kirby 2002; George et al., 2006; Heinstrom 2002; Jiao e 


\section{CONGRESSO ISKO Espanha - Portugal}

Onwuegbuzie 1997; 1999; Jiao et al. 2006, Jiao et al. 1996; Mellon 1988; Onwuegbuzie 1997; Onwuegbuzie e Jiao 1998; 2004; Wang e Soergel, 1998)”.

É preciso atentar para o fato de que não é possível mais se limitar à tarefa de localizar fontes de informação. É necessário levar em consideração tarefas de interpretação, formulação e aprendizagem envolvidas no processo de busca dessa informação. O aumento no acesso à vasta quantidade de informação requer, entretanto, serviços que se centrem no significado da busca mais do que meramente na localização da fonte. Nessa perspectiva, os usuários da informação não podem ser vistos apenas como integrantes do sistema, mas como a "razão de ser" do serviço de informação.

Sistemas de informação organizados na perspectiva tradicional concentram-se prioritariamente na aquisição e administração de grandes coleções de materiais. Assumiu-se, durante décadas, que as atividades técnicas dos sistemas eram o seu ponto estratégico. Considerava-se que os usuários utilizavam o sistema exatamente da maneira como estes tinham sido projetados. Não se imaginava indagar, aos sistemas, questões imprescindíveis sobre a identidade e propósitos principais de seus usuários. Como a informação era considerada algo existente fora das pessoas e passível de ser transferida de uma para outra, parecia ser possível que eficiência e sucesso das operações de um sistema pudessem ser medidos em função do número de fontes de informações recuperadas pelo sistema versus o que realmente foi de interesse do usuário. Isso, na realidade, coloca novamente o usuário como um processador imperfeito da informação, pois é já sabido que nem todas as pessoas se interessam pelas mesmas fontes indicadas. Resulta desse procedimento que hoje em dia se conhece muita coisa sobre planejamento, aquisição, organização, controle e desenvolvimento de coleções, mas muito pouco sobre como as pessoas fazem uso dos sistemas ou para que fins e como a informação, a matéria-prima dos sistemas, está sendo utilizada (Ferreira 1995).

\section{A ORGANIZAÇÃO DA INFORMAÇÃO NA WEB DE DADOS}

O impacto do uso da Web na sociedade, nos indivíduos e nas organizações tornou-se objeto de pesquisa, extrapolando o campo especializado da computação aplicada, e atingindo áreas de estudos organizacionais e sociológicos. Por ser essencialmente dinâmica e sem fronteiras, tanto do ponto de vista físico como virtual, é importante que seja conhecida em detalhes, tanto para assegurar sua livre transformação quanto para permitir sua disponibilidade, confiabilidade e acessibilidade por todos.

A denominada Web 2.0 segue uma filosofia com princípios de leitura e escrita de natureza participativa, em que cada usuário pode intervir diretamente na escolha e introdução de dados no âmbito de cada site; é cooperativa, uma vez que compartilha ideias, preferências, informações e conhecimento; é interativa, na medida em que, através de toda a gama de recursos multimídia, é possível um diálogo simultâneo com os usuários; é democrática, pois sob essa filosofia existe liberdade de expressão, de pensamento, e, sobretudo, de trânsito de informações, independentemente dos interesses de cada um; é também sociotécnica, pois, através de todas as suas características, é possível um intercâmbio de culturas, religiões, etnias e outros. Hoje, não só o usuário participa como também adiciona conteúdo que ao serem compartilhados em outros recursos colaborativos passam a receber comentários dos membros vinculados a sua comunidade agregando valor à informação compartilhada, além de criar vários caminhos para a localização do próprio recurso. Assim, temos uma nova relação e interação na qual aumenta o nível sofisticado de atitudes sociais eletrônicas. 


\section{CONGRESSO ISKO Espanha - Portugal}

A Web Semântica apresenta um novo modelo de interatividade entre o usuário da informação e a internet. Esta deve idealmente apresentar toda sua informação rotulada com indicações de contexto e significado, organizada de forma que tanto humanos como máquinas possam entendê-la. Surge uma nova denominação para diferentes evoluções pretendidas na Web e na interação desta com o usuário: a Web 3.0 (Passarelli 2008). Entre os muitos aspectos incluídos nessa denominação, destacam-se:

- Web semântica;

- Web geoespacial;

- Web 3D e de realidade aumentada;

- Conteúdo Web mais dinâmico, agregando inteligência artificial;

- Maior compatibilidade da Web com a tecnologia de banco de dados;

Neste ambiente contemporâneo, surge um novo usuário da informação com novas demandas por recursos computacionais e novas capacidades em produzir novos conhecimentos. Considerando-se os principais aspectos da Competência Informacional: a Tecnologia da Informação, as Fontes de Informação, os Processos de Informação, o Controle da Informação, a Construção do Conhecimento, a Extensão do Conhecimento e a Inteligência (Bruce 2003), destaca-se um profissional ativamente envolvido com os processos de ensino e aprendizado em Competência Informacional: o bibliotecário (Souza 2010).

\section{PROCEDIMENTOS METODOLÓGICOS}

Esta pesquisa de caráter qualitativo e exploratório tem como objetivo proporcionar uma reflexão sobre a atuação do profissional da informação na organização do conhecimento na web de dados, com vistas a torná-lo mais explícito ou a construir hipóteses. A pesquisa preocupa-se, portanto, com aspectos de realidade que não pode ser quantificada, centrando-se na compreensão e explicação da dinâmica das relações sociais. A revisão da literatura apresenta um panorama da educação com os novos recursos informacionais e digitais, as literacias da informação, a transdisciplinaridade entre o campo da Biblioteconomia, Informação e Comunicação e a crescente tendência das Escolas de Informação iSchools. Os procedimentos metodológicos adotados para este trabalho compreendem duas dimensões: teórica e aplicada. Do ponto de vista teórico, caracteriza-se como sendo do tipo descritiva exploratória de natureza qualitativa, devido ao fato de ter como objetivo a reunião de dados e informações sobre os temas abordados.

\section{RESULTADOS}

Saberes científicos e o acesso a inovações tecnológicas apresentam-se distribuídos de forma muito desigual, considerando-se nesta comparação países, regiões, estratos sociais, faixas etárias, níveis educacionais, dentre outros. Assim, a problemática da diversidade cultural e os estudos sobre ela devem fazer parte da consideração teórica, da investigação empírica e do planejamento de políticas na área de fomento à Competência Informacional. Além disso, o usuário da informação típico não deseja apenas extrair informações específicas e definitivas no menor tempo possível ou, alternativamente, se dispõe a investir esforço para buscar e explorar informação e conhecimento. A verdade é que as pessoas oscilam continuamente entre extrair e explorar, e o uso da informação é um processo confuso, desordenado, sujeito aos caprichos da natureza humana, como qualquer outra atividade (Canclini 2009). Entre os 


\section{CONGRESSO ISKO Espanha - Portugal}

elementos mais importantes que influenciam o uso da informação estão as atitudes do indivíduo em relação à informação e a sua busca, atitudes essas que são fruto da educação, do treinamento, da experiência passada, das preferências pessoais.

O universo da informação digital se expande no contexto do que hoje chamamos de Big Data, onde a busca por informações fica cada vez mais difícil uma vez que o universo digital está constituído por dados não estruturados que precisam ser organizados, acessados, apropriados e então utilizados na produção de novos conhecimentos. A Web de Dados virá com o objetivo de organizar estas informações para que os usuários tenham mais facilidade na busca da informação. A busca Semântica como é chamada os buscadores da Web de Dados, organizam informações por assuntos determinados, conectando conhecimentos. A busca Semântica da Web de Dados dividirá os resultados em categorias para que a busca da informação seja mais rápida e organizada.

A Figura 1 relaciona o Grau de Conectividade da Informação com o Grau de Conectividade Social de acordo com a evolução tecnológica: Web - Conectando Informação, Web Social - Conectando Pessoas, Web Semântica - Conectando Conhecimento e a Metaweb - Conectando Inteligência.

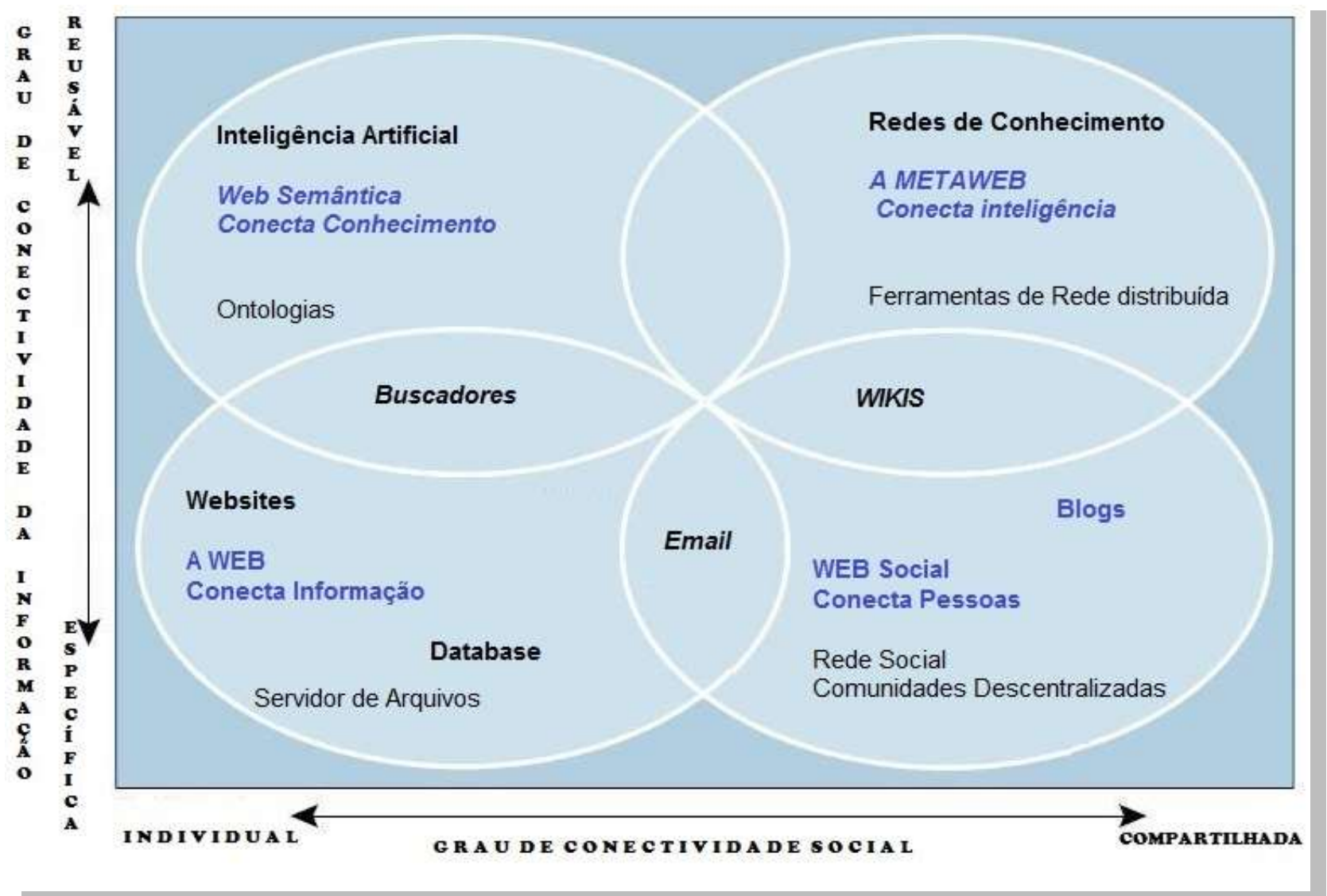

Figura 1. Evolução Tecnológica da Web - Adaptação do Autor

Fonte: http://www.novaspivack.com/science/new-version-of-my-metaweb-graph-the-future-of-the-net 


\section{CONGRESSO ISKO Espanha - Portugal}

A organização do conhecimento liga os três processos de uso estratégico da informação - a criação de significado, a construção do conhecimento e a tomada de decisões - num ciclo contínuo de aprendizagem e adaptação que podemos chamar de ciclo do conhecimento.

Bibliotecas estão desenvolvendo continuamente processos e serviços de forma a explorar os benefícios da Web 2.0. Library 3.0 refere-se a bibliotecas utilizando tecnologias como a Web Semântica, a computação em nuvem, dispositivos móveis, e re-imaginando o uso de tecnologias estabelecidas, tais como pesquisa federada (RFID), para facilitar a disseminação do conteúdo gerado pelo usuário e a colaboração para promover e fazer coleções de bibliotecas acessíveis. O resultado final da Biblioteca 3.0 é a expansão da "biblioteca sem fronteiras", onde as coleções podem ser feitas facilmente disponíveis aos usuários da biblioteca, independentemente da sua localização física. Biblioteca 3.0 é um complemento virtual para os espaços físicos da biblioteca (Belling 2013).

É neste contexto de crescente disponibilidade de dados que a "era digital" se apresenta com múltiplas oportunidades de atuação para o profissional da informação no uso das tecnologias da informação e comunicação na gestão e organização da informação na Web de Dados.

\section{CONCLUSÕES}

Há atualmente a necessidade de novas propostas para abordagens gerenciais e estratégicas associadas à utilização da Tecnologia da Informação na apropriação e geração de conhecimento no contexto da Web Inteligente.

Diante da emergência de uma economia digital - formação de padrões complexos a partir de uma multiplicidade de interações, cumpre-nos avaliar as contínuas tensões na educação entre a Ciência da Informação e a Biblioteconomia neste novo cenário orientado à formação de recursos humanos: futuros bibliotecários - profissionais da informação. É preciso preparar profissionais da informação habilitados à lidar com a complexidade do mercado de trabalho global, proporcionando uma revisão sistemática nos planos de ensino associada às principais questões que estão à mudar em sua área de conhecimento no contexto social, político, econômico, tecnológico e cultural: o impacto dos dispositivos digitais e redes sociais, a evolução dos serviços bibliotecários incluindo referência virtual, o suporte ubíquo a bibliotecas, acesso digital a repositórios, preservação digital, os novos esforços para organizar o conhecimento, a Web Semântica, o catálogo da biblioteca de próxima geração, o impacto da publicação digital e dos e-books, as questões políticas relacionadas com o acesso em banda larga e a neutralidade da rede, novas interpretações de direitos autorais relacionados com a digitalização em massa de livros e artigos acadêmicos, novas iniciativas para integrar bibliotecas, arquivos e museus (RUBIN, 2010).

O desafio imposto pela Era Digital em entender o papel da informação, e em particular na área da Ciência da Informação, no cenário que os especialistas chamam de a "4 ${ }^{\circ}$ Revolução Industrial" - iniciada no início da década de $90 \mathrm{com}$ ascensão da desmaterialização de funções e processos e do trabalho do conhecimento. A formação e capacitação de recursos humanos, cooperação tecnológica, uso em larga escala das tecnologias da informação e comunicação TICs estão a pavimentar o caminho da revolução digital.

$\mathrm{Na}$ sociedade em contínua mudança, temos que repensar os modelos de ensino e observar as complexidades do mercado de trabalho 4.0 e suas relações com a Informação, Comunicação e Cultura e 


\section{CONGRESSO ISKO Espanha - Portugal}

estruturar programas que permitam formar o profissional da informação com as competências necessárias para atuar em um mercado de trabalho cada vez mais dependente das competências digitais.

$\mathrm{Na}$ Era da Informação e, mais precisamente, na conjuntura de rede onde estamos imersos, muitos são os desafios e os problemas que não podemos apenas enfrentar, mas devemos estudar com minúcia e profundidade a fim de irmos encontrando as respostas globais e específicas mais adequadas (SILVA, 2008).

Neste trabalho, discutimos as tendências nesse campo, em especial interesse aquelas que influenciam o contexto da chamada Biblioteca 3.0, de modo que, cada vez mais, a tecnologia possa proporcionar a criação e obtenção de valor ao usuário da informação. Espera-se que o desenvolvimento dessa área leve a um maior entendimento dos recursos da tecnologia colaborativa utilizados em ambientes informacionais digitais. Em qualquer caso, a inserção dessas tecnologias consiste em inovação que deve estar vinculada à tradição e à missão das bibliotecas e dos repositórios bem como na atuação do profissional da informação na organização e gestão da informação na Web de Dados.

\section{REFERÊNCIAS}

Belling, A. (2013). Exploring Library 3.0 and beyond. Retrieved 18 June, 2017 from http://www.libraries.vic.gov.au/downloads/20102011_Shared_Leadership_Program_Presentation_Day_/e xploring_library_3.pdf

Bilal, D. (2000). Children's use of the Yahooligans! Web search engine: I. Cognitive, physical and affective behaviors on fact-based search tasks. Journal of the American Society for Information Science, 51(7), 646-665.

Bilal, D. (2001). Children's use of the Yahooligans! Web search engine: II. Cognitive and physical behaviors on research tasks. Journal of the American Society for Information Science and Technology, 52(2), 118-136.

Bilal, D. (2002). Children's use of the Yahooligans! Web search engine: III. Cognitive and physical behaviors on fully self-generated search tasks. Journal of the American Society for Information Science and Technology, 53(13), 1170-1183.

Bilal, D. \& Kirby, J. (2002). Differences and similarities in information seeking: children and adults as Web users. Information Processing and Management, 38(5), 649-670

Bruce, C. (2003). Seven Faces of Information Literacy. Retrieved 18 June 2017 from http://www.bestlibrary.org/digital/files/bruce.pdf

Canclini, N, G. (2009). Diferentes, desiguais e desconectados. Rio de Janeiro: Editora UFRJ. ISBN $857108291 \mathrm{X}$

CGI.br (2011). Dimensões e características da WEB brasileira: um estudo do .gov.br. http://www.cgi.br/media/docs/publicacoes/2/cgibr-nicbr-censoweb-govbr-2010.pdf 


\section{CONGRESSO ISKO Espanha - Portugal}

Cole, C. (1997). Information as process: The difference between corroborating evidence and "information" in humanistic research domains. Information Processing and Management, 33(1), 55-67.

Dudziak, E, A. (2001). A Information Literacy e o papel educacional das bibliotecas. Dissertação (Mestrado) - Escola de Comunicações e Artes, Universidade de São Paulo, São Paulo, 2001.

Dudziak, E, A. (2003). "Information literacy: princípios, filosofia e prática". Ciência da Informação, Brasília, v.32, n.1, p. 23-35.

Ferreira, S, M, S, P. (1995). "Novos paradigmas e novos usuários da informação”. Ciência da Informação, Brasília, v.25, n.2, p. 217-223.

George, C., Bright, A., Hurlbert, T., Linke, E. C., St. Clair, G. \& Stein, J. (2006). Scholarly use of information: graduate students' information seeking behaviour. Information Research, 11(4), paper 272. Retrieved 18 June, 2017 from http://informationr.net/ir/11-4/paper272.html (Archived by WebCite ${ }^{\circledR}$ at http://www.webcitation.org/5cbDFityo)

Harada, V. H. (2002). Personalizing the information search process: A case study journal writing with elementary-age students. School Library Media Research, 5. Retrieved 18 June, 2017from http://www.ala.org/aasl/sites/ala.org.aasl/files/content/aaslpubsandjournals/slr/vol5/SLMR_PersonalizingI nfoSearch_V5.pdf (Archived by WebCite@ at http://www.webcitation.org/5cbDWgyrj)

Heinström, J. (2002). Fast surfers, broad scanners and deep divers - personality and information seeking behaviour. Åbo (Turku), Finland: Åbo Akademi University Press. (Doctoral dissertation.) Retrieved 18 June, 2017 from http://www.abo.fi/fakultet/media/21373/thesis_heinstrom.pdf

Jiao, Q. G. \& Onwuegbuzie, A. J. (1997). Antecedents of library anxiety. The Library Quarterly, 67(4), 372-389.

Jiao, Q. G. \& Onwuegbuzie, A. J. (1999). Identifying library anxiety through students' learning modality preferences. Library Quarterly, 69(2), 202-216.

Jiao, Q. G., Onwuegbuzie, A. J. \& Bostick, S. L. (2006). The relationship between race and library anxiety among graduate students: a replication study. Information Processing and Management, 42(3), 843-851.

Jiao, Q. G., Onwuegbuzie, A. J. \& Lichtenstein, A. (1996). Library anxiety: characteristics of 'at-risk' college students. Library and Information Science Research, 18(2), 151-163.

Mellon, C.A. (1988). Attitudes: the forgotten dimension in library instruction. Library Journal, 113(14), 137-139.

Onwuegbuzie, A.J. (1997). Writing a research proposal: the role of library anxiety, statistics anxiety and composition anxiety. Library and Information Science Research, 19(1), 5-33. 


\section{CONGRESSO ISKO Espanha - Portugal}

Onwuegbuzie, A. J. \& Jiao, Q. G. (1998). The relationship between library anxiety and learning styles among graduate students: Implications for library instruction. Library and Information Science Research, 20(3), 235-249.

Onwuegbuzie, A. J. \& Jiao, Q. G. (2004). Information search performance and research achievement: an empirical test of the Anxiety-Expectation Mediation model of library anxiety. Journal of the American Society for Information Science and Technology, 55(1), 41-54.

Passarelli, B. (2008). "Do Mundaneum à WEB Semântica: discussão sobre a revolução nos conceitos de autor e autoridade das fontes de informação". DataGramaZero - Revista de Ciência da Informação - v.9 n.5, P.1-13.

Passarelli, B.; Junqueira, A, H. Gerações Interativas Brasil. Crianças e Adolescentes Diante das Telas. http://ccvap.futuro.usp.br/gerinter2012.pdf

Pitts, J. M. (1995). Mental models of information. The 1993-1994 AASL/Highsmith Research Award Study. School Library Media Quarterly, 23(3), 177-184.

RUBIN, R. (2010). Foundations of library and information science. New York: Neal Schuman.

Serola, S. \& Vakkari, P. (2005). The anticipated and assessed contribution of information types in references retrieved for preparing a research proposal. Journal of the American Society for Information Science and Technology, 56(4), 373-381.

SILVA, Armando Malheiro da (2008). Inclusão digital e literacia informacional em ciência da informação. Prisma.Com, Porto, 7 (Jul.).p.16-43. ISSN: 1646-3153. Url: http://revistas.ua.pt/index.php/prismacom/article/view/683/pdf

Sousa, R, S, C., Nascimento, B, S. (2010). "Competências Informacionais: uma análise focada no currículo e na produção docente dos cursos de Biblioteconomia e gestão da informação". Revista ACB: Biblioteconomia em Santa Catarina, Florianópolis, v.15, n.2, p.130-150.

Tang, R. \& Solomon, P. (1998). Toward an understanding of the dynamics of relevance judgment: an analysis of one person's search behavior. Information Processing and Management, 34(2), 237-256.

UNESCO. (2017). Building Knowledge Societies. Retrieved March 20, 2017, from http://en.unesco.org/themes/building-knowledge-societies

Vakkari, P. (2001). A theory of the task-based information retrieval process: a summary and generalisation of a longitudinal study. Journal of Documentation, 57(1), 44-60.

Vakkari, P. \& Hakala, N. (2000). Changes in relevance criteria and problem stages in task performance. Journal of Documentation, 56(5), 540-562.

Vakkari, P., Pennanen, M. \& Serola, S. (2003). Changes of search terms and tactics while writing a research proposal: a longitudinal case study. Information Processing and Management, 39(3), 445-463. 


\section{CONGRESSO ISKO Espanha - Portugal}

Wang, P. \& Soergel, D. (1998). A cognitive model of document use during a research project. Study 1. Document selection. Journal of the American Society for Information Science, 49(2), 115-133.

W3C Brasil (2011). Missão do W3C. http://www.w3c.br/Sobre/MissaoW3C

Yang, S. (1997). Information seeking as problem-solving using a qualitative approach to uncover the novice learners' information-seeking process in a perseus hypertext system. Library and Information Science Research, 19(1), 71-92.

Kuhlthau, C.C., HeinstrÖm, J. \& Todd, R.J. (2008). "The 'information search process' revisited: is the model still useful?" Information Research, 13(4) paper 355. Retrieved March 20, 2017, from http://InformationR.net/ir/13-4/paper355.html

ACKNOWLEDGMENT: FAPESP Research Project - Processo 2016/07358-6 